\title{
Branching rules for the subgroups of the unitary group ${ }^{a)}$
}

\author{
D. Braunschweig and K. T. Hecht
}

Department of Physics, University of Michigan, Ann Arbor, Michigan 48109

(Received 31 August 1977)

Expressions are given in terms of simple matrices $d$ for the reduction of the Kronecker (outer) product of two or more irreducible representations which can be characterized by Young patterns. These are then used to obtain practical formulas for branching rules. The needed matrices $d$ can be constructed by a very efficient recursive process.

\section{INTRODUCTION}

The effective use of group theoretical methods in atomic and nuclear shell model calculations requires that the many-particle states be classified according to the irreducible representations (IR) of the unitary group and its subgroups. ${ }^{1}$ These IR may conveniently be labeled by their permutation symmetry in terms of Young patterns. ${ }^{2}$ The knowledge of the relevant branching rules appropriate to the subgroup chain under consideration is usually needed at some step in the calculations.

Therefore, it is not surprising that considerable effort has been expended in finding practical ways of determining these branching rules, and numerous tabulations are available. ${ }^{3}$ The plethysm of $S$ functions has been used to obtain branching rules, ${ }^{4}$ but except for simple cases the procedure becomes cubersome. ${ }^{5}$

The problem of calculating branching rules and the resolution of the Kronecker (outer) product can be simplified greatly if advantage is taken of the systematic decomposition of Young patterns into their completely symmetric or antisymmetric components. The simplicity gained ther eby allows us to obtain the resolution of the Kronecker (outer) product of more than two patterns directly, which becomes an essential part in the evaluation of branching rules.

Our method is applicable to any set of subgroups whose IR can be labeled by Young patterns, and furthermore it is well suited for desk top calculations.

\section{NOTATION}

We denote by $[\lambda]^{n}$ the partition

$$
\lambda_{1}+\lambda_{2}+\cdots+\lambda_{n}=n, \quad \lambda_{1} \geqslant \lambda_{2} \geqslant \cdots \geqslant \lambda_{n} \geqslant 0
$$

of the integer $n$. With this partition $[\lambda]^{n}$ we associate in the usual manner ${ }^{7}$ a Young pattern with $\lambda_{1}$ boxes in the first row, $\lambda_{2}$ boxes in the second row,..., etc. We say that a partition $[\mu]^{n}$ is of lower symmetry than $[\lambda]^{n}$ if the first nonzero difference $\left(\mu_{t}-\lambda_{t}\right)$ is negative. This allows us to order the $p(n)$ partitions of $n$ in order of decreasing symmetry, such that if $i>j$, than $[\lambda]_{i}^{n}$ is of lower symmetry than $[\lambda]_{j}^{n}$. In what follows we denote by $\lambda_{s i}^{n}$ the number of boxes in row $s$ of partition number $i$.

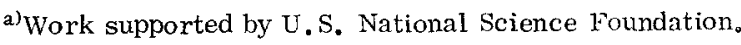

We denote by $[\tilde{\lambda}]_{i}^{n}$ the partition conjugate of $[\lambda]_{i}^{n}$ obtained from $[\lambda]_{i}^{n}$ by inter changing rows and columns.

\section{DECOMPOSITION OF PATTERNS}

In this section we introduce the decomposition of patterns into their completely symmetric or antisymmetric components.

With each pattern $[\lambda]_{i}^{n}$ we associate an antisymmetric factor pattern $A\left([\lambda]_{i}^{n}\right)$ such that

$$
A\left([\lambda]_{i}^{n}\right)=\left[1^{\tilde{\lambda}_{1 i}}\right] \times\left[1^{\tilde{\lambda}_{2 i}}\right] \times \cdots \times\left[1^{\tilde{\lambda}_{\lambda_{1} i}}\right],
$$

For example, if $[\lambda]_{i}^{n}=[43221]$, then

$$
A\left([\lambda]_{i}^{n}\right)=A(\bigoplus)=\mathrm{B} \times \mathrm{B} \times \mathrm{G} \times \square .
$$

The products on the right are the Kronecker (outer) products of pattern multiplication which can be evaluated using Littlewood's rules. ${ }^{7}$

Given a pattern $[\lambda]_{i}^{n}$ we may set up the system of linear equations

$$
A\left([\lambda]_{j}^{n}\right)=\sum_{k=i}^{p(n)} d_{j k}^{n \wedge}[\lambda]_{k}^{n}, j=i, i+1, \ldots, p(n),
$$

or inversely,

$$
[\lambda]_{k}^{n}=\sum_{j=i}^{p(n)} c_{k j}^{n A} A\left([\lambda]_{j}^{n}\right), k=i, i+1, \ldots, p(n),
$$

where

$$
c_{k j}^{n A}=(-)^{k+j}\left|d_{j k}^{n A}\right|
$$

is the cofactor of the matrix element $d_{j k}^{\text {nd }}$.

As an example we give in Fig. 1 the matrices $d_{j k}^{n S}$ and $c_{k j}^{n S}$ for $n=5$.

In complete analogy with the above we can associate with each pattern $[\lambda]_{i}^{n}$ a symmetric factor pattern $S\left([\lambda]_{i}^{n}\right)$ such that

$$
S\left([\lambda]_{i}^{n}\right)=\left[\lambda_{1 i}\right] \times\left[\lambda_{2 i}\right] \times \cdots \times\left[\lambda_{n i}\right] .
$$

For example, if $\mid \lambda]_{i}^{n}=[43221]$, then

$$
S\left([\lambda]_{i}^{n}\right)=S(\bigoplus)=\square \times \square \times \square \times \square \times \square .
$$

Again we may set up the system of equations

$$
S\left([\lambda]_{j}^{n}\right)=\sum_{k=1}^{i} d_{j k}^{n S}[\lambda]_{k}^{n}, \quad j=1,2, \ldots, i,
$$

or inversely, 


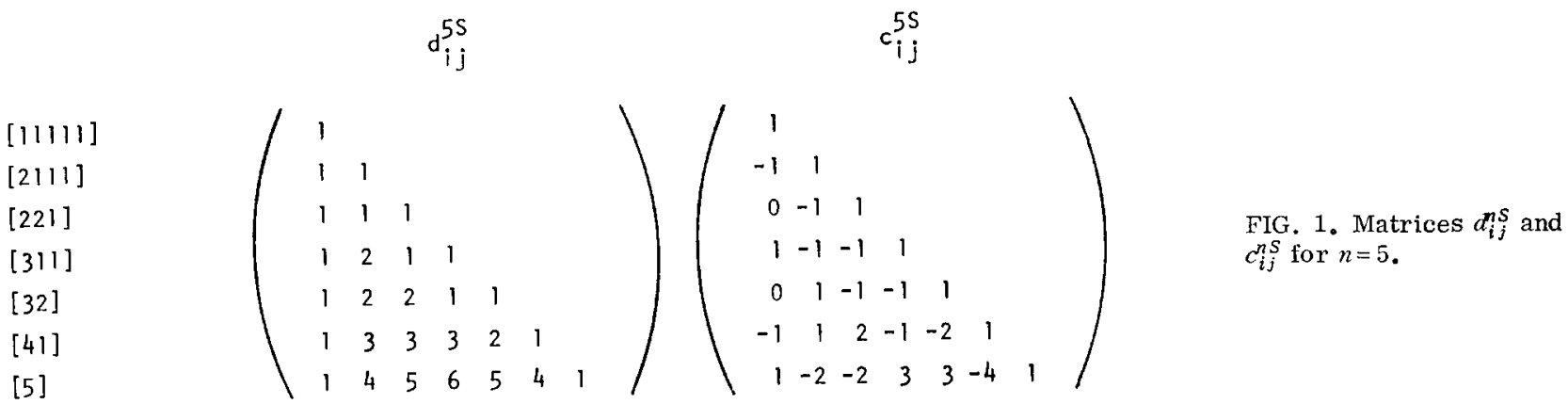

$$
[\lambda]_{k}^{n}=\sum_{j=1}^{i} c_{k j}^{n S} S\left([\lambda]_{j}^{n}\right), \quad k=1,2, \ldots, i .
$$

If we adhere to the convention that whenever we use antisymmetric decomposition we order the patterns in order of decreasing symmetry, while when using symmetric decomposition we order them in order of increasing symmetry, we obtain the result

$$
c_{k j}^{n A}=c_{k j}^{n S} \text {, and } d_{j k}^{n A}=d_{j k}^{n S} \text {, }
$$

which allows us to drop the superscripts $S$ and $A$ from here on.

Since all our results are expressed in terms of the matrices $c_{i j}$ and $d_{i j}$ we summarize in the Appendix some of their properties and giverds well a recursion relation for the matrix $d_{i j}$.

\section{RESOLUTION OF THE KRONECKER (OUTER) PRODUCT}

For arbitrary $[\lambda]_{i}^{n}$ and $[\lambda]_{j}^{m}$ we have

$$
[\lambda]_{i}^{n} \times[\lambda]_{j}^{m}=\left(\sum_{k=i}^{p(n)} c_{i k}^{n} A\left([\lambda]_{k}^{n}\right)\right) \times\left(\sum_{i=j}^{p(m)} c_{j l}^{m} A\left([\lambda]_{l}^{m}\right)\right) .
$$

The $\lambda_{1 k}+\lambda_{1 l}$ factors in the product

$$
\left[1^{\tilde{\lambda}_{1 k}}\right] \times \cdots \times\left[1^{\tilde{\lambda}_{\lambda_{1} k}}\right] \times\left[1^{\tilde{\lambda}_{1 l}}\right] \times \cdots \times\left[1^{\tilde{\lambda}_{\lambda_{1} l}}\right]
$$

result from the decomposition of the pattern $[\lambda]_{p}^{n+m}$, where

$$
\lambda_{s \uparrow}^{n+m}=\lambda_{s k}^{n}+\lambda_{s l}^{m}, \quad s=1,2, \ldots, \max \left(\tilde{\lambda}_{1_{k}}^{n}, \tilde{\lambda}_{1_{l}}^{m}\right) .
$$

Therefore,

$$
\begin{aligned}
{[\lambda]_{i}^{n} \times[\lambda]_{j}^{m} } & =\sum_{k, l} c_{i k}^{n} c_{j l}^{m} A\left([\lambda]_{p}^{n+m}\right) \\
& =\sum_{k, l, q} c_{i k}^{n} c_{j l}^{m} d_{p, q}^{n+m}[\lambda]_{q}^{n+m} \\
& =\sum_{q} R_{i, j}^{\natural}[\lambda]_{q}^{n+m},
\end{aligned}
$$

where

$$
R_{i, j}^{\mathbf{q}}=\sum_{\substack{k=1, i \\ l=1, j}} c_{i k}^{n} c_{j l}^{m} d_{p, q}^{n+m}
$$

gives the number of times the pattern $[\lambda]_{q}^{n+m}$ is contained in $[\lambda]_{i}^{n} \times[\lambda]_{j}^{m}$.

This result can be easily generalized to the case where there are more than two factors in the product,

$$
\begin{aligned}
& {[\lambda]_{i_{1}}^{n_{1}} \times[\lambda]_{i_{2}}^{n_{2}} \times \cdots \times[\lambda]_{i_{f}}^{n_{f}}} \\
& \quad=\sum_{q} R_{i_{1} i_{2}}^{q} \cdots i_{f}[\lambda]_{q}^{n_{1}+n_{2}+\cdots+n_{f}},
\end{aligned}
$$

where

$$
\begin{aligned}
& R_{i_{1} i_{2} \cdots i_{f}}^{q} \\
& \quad=\sum_{k_{1} k_{2} \cdots k_{f}} c_{i_{1} k_{1}}^{n_{1}} c_{i_{2} k_{2}}^{n_{2}} \cdots c_{i_{f} k_{f}}^{n_{f}} d_{p q}^{n_{1}+n_{2}+\cdots+n_{f}} .
\end{aligned}
$$

It should be noted that the use of Eq. (18) for $R_{i_{1} i_{2} \cdots i_{f}}$ involves considerably less computation than the repeated use of Eq. (16) if the number of factors in Eq. (17) is greater than two.

\section{BRANCHING RULES}

The use of canonical subgroup chains greatly simplifies the calculation of branching rules. For example, the branching rules for the chain

$$
\mathrm{U}(N) \supset \mathrm{U}(N-1) \supset \cdots \supset \mathrm{U}(1)
$$

are given by the "betweenness" conditions ${ }^{8}$ of Weyl's branching theorem. However the physically relevant subgroup chains are determined by the symmetries of the physical problem and seldom coincide with a canonical chain. Therefore, we consider below the branching rule problem for an arbitrary subgroup chain.

Let $G$ and $H$ be two arbitrary subgroups of the full linear group such that $G \supset H$. To obtain the branching rules for $G \supset H$ we shall assume that the IR's of $H$ contained in the totally antisymmetric IR of $G$ are known; that is, we assume that the coefficient $\alpha_{s h}$ are known in

$$
\left[1^{s}\right]=\sum_{h} \alpha_{s h}[\lambda]_{h}^{\prime},
$$

where $\left[1^{s}\right]$ is a totally antisymmetric IR of $G$ and $[\lambda]_{h}^{\prime}$ are IR of $H$.

$$
\begin{aligned}
& \text { Then } \\
& \begin{aligned}
{[\lambda]_{i}^{n}=} & \sum_{j=i}^{(n)} c_{i j}^{n} A\left([\lambda]_{j}^{n}\right) \\
= & \sum_{j} c_{i j}^{n}\left[1^{\tilde{\lambda}_{1 j}}\right] \times\left[\tilde{1}^{\tilde{\lambda}_{2 j}}\right] \times \cdots \times\left[1^{\left.\tilde{\lambda}_{\lambda_{1} j}\right]}\right. \\
= & \sum_{j} c_{i j}^{n} \sum_{h_{1} h_{2} \cdots h_{h_{1}}} \alpha_{{\tilde{\lambda_{1 j}}}_{h_{1}}} \cdots \alpha_{\tilde{\lambda}_{\lambda_{1}{ }^{j} h_{\lambda_{1}}}}[\lambda]_{h_{1}}^{\prime} \\
& \times \cdots \times[\lambda]_{h_{\lambda_{1}}}^{\prime} .
\end{aligned}
\end{aligned}
$$


Introducing Eq. (17) into Eq. (21) we get

$$
[\lambda]_{i}^{n}=\sum_{q} S_{i}^{q}[\mu]_{q}^{\prime} \text {, }
$$

where

$$
S_{i}^{q}=\sum_{h_{1} h_{2} \cdots h_{\lambda_{1}}} c_{i j}^{n} \tilde{\alpha}_{\lambda_{1 j} h_{1}} \cdots \alpha_{\lambda_{\lambda_{1} j^{j}} h_{1}} R_{h_{1} h_{2} \cdots h_{\lambda_{1}}}^{a}
$$

gives the number of times the IR $[\mu]_{q}^{\prime}$ of $H$ is contained in the $\operatorname{IR}[\lambda]_{i}^{n}$ of $G$.

In general, Eq. (22) can be simplifed further by using modification rules appropriate to the subgroup $H$.

\section{SPECIAL CASES}

\section{A. Two column patterns}

Assume that $[\lambda]_{i}^{n}$ is such athat $\lambda_{s i}^{n}=0$ for $s>2$ while $\tilde{\lambda}_{1 i}^{n} \neq 0 \neq \tilde{\lambda}_{2 i}^{n}$. It is clear that in this case

$$
d_{i j}^{n}=0 \text { for } j>i \text { and } d_{i j}^{n}=1 \text { for } j \leqslant i,
$$

which in turn means that

$$
c_{i j}^{n}=(-)^{i+j}\left|d_{j i}^{n}\right|=\delta_{i j}-\delta_{i+1 j} \text {. }
$$

Introducing this into Eq. (5) we get

$$
[\lambda]_{i}^{n}=\left[1^{\tilde{\lambda}_{1 i}}\right] \times\left[1^{\tilde{\lambda}_{2 i}}\right]-\left[1^{\tilde{\lambda}_{1 i}+1}\right] \times\left[1^{\tilde{\lambda}_{2 i}^{-1}}\right] .
$$

This formula is useful in atomic spectroscopy where the electron being a fermion of spin $\frac{1}{2}$ requires that the spatial symmetry of a many-electron wavefunction be described by a two-columned pattern.

\section{B. Two-rowed patterns}

Assume that $[\lambda]_{i}^{n}$ is such that $\lambda_{s i}^{n}=0$ for $s>2$ while $\lambda_{1 i}^{n} \neq 0 \neq \lambda_{2 i}^{n}$. It is clear that for this case Eqs. (24) and (25) hold. Introducing Eq. (25) into Eq. (10) we get

$$
[\lambda]_{i}^{n}=\left[\lambda_{1 i}\right] \times\left[\lambda_{2 i}\right]-\left[\lambda_{1 i}+1\right] \times\left[\lambda_{2 i}-1\right] .
$$

This formula is useful when dealing with SU(3) since all the patterns in this case are at most two rowed. In Elliot's language ${ }^{9}$

$$
(\lambda \mu)=(\lambda+\mu, 0) \times(\mu, 0)-(\lambda+\mu+1,0) \times(\mu-1,0) .
$$

The product of two $\mathrm{SU}(3)$ representations is easily expressed in terms of the appropriate $d$ matrices,

$$
\begin{aligned}
R_{i, j} & =\sum_{\substack{k=1, i \\
i=1, j}} c_{i k}^{n} c_{j l}^{m} d_{p+q}^{n+m} \\
& =\sum_{k, l}\left(\delta_{i k}-\delta_{i+1, k}\right)\left(\delta_{j l}-\delta_{j+1, l}\right) d_{p, q}^{n+m} \\
& =d_{p(i, j)_{q}}^{n+m}+d_{p(i+1, j+1)_{q}}^{n+m}-d_{p(i+1, j)_{q}}^{n+m}-d_{p(i, j+1)_{q}}^{n+m} .
\end{aligned}
$$

\section{APPENDIX}

We summarize below the main properties of the matrices $c_{i j}^{n}$ and $d_{i j}^{n}$ :

(1) $c_{i j}^{n}=(-)^{i+j}\left|d_{j i}^{n}\right|$,

(2) $d_{i j}^{n}=c_{i j}^{n}=0$ for $j>i$,

(3) $d_{i i}^{n}=c_{i i}^{n}=1$ for $i=1, \ldots, p(n)$,
(4) $d_{i j}^{n} \geqslant 0$ for $i, j=1, \ldots, p(n)$,

(5) $\operatorname{Tr}(D)=\operatorname{Tr}(C)=p(n)$,

(6) $\operatorname{Det}(D)=\operatorname{Det}(C)=1$,

(7) $\sum_{j=1} c_{i j}^{n}=0$ for $i=1,2, \ldots,[p(n)-1]$,

(8) both the matrix elements $c_{i j}^{n}$ and $d_{i j}^{n}$ are integers.

The matrix $d_{i j}^{n}$ may be obtained directly from its definition in Eqs. (4) and (9); however, in practice it is more convenient to build the necessary matrix elements from the recursion relation we give below. This is particularly important for the matrix $d_{i j}^{n}$ since normally only a few matrix elements are needed.

Let $[\lambda]_{i}^{n}$ and $[\lambda]_{j}^{m}$ be partitions of the integers $n$ and $m$ respectively, with say $n>m$. If

$$
\left(\lambda_{s i}^{n}-\lambda_{s j}^{m}\right) \geqslant 0, s=1, \ldots, n .
$$

then the set of positive integers $\left(\lambda_{s i}^{n}-\lambda_{s j}^{m}\right)$ form a partition of the integer $n-m$ which we denote by $\left[\lambda_{i}-\lambda_{j}\right]^{n-m}$. For $n-m=3$, e.g. , the numbers $\lambda_{s i}^{n}-\lambda_{s j}^{m}=210000$ or 020010 both define the same partition $[21]_{k=2}^{3}$. We define

$$
H\left([\lambda]_{i}^{n},[\lambda]_{j}^{m},[\lambda]_{k}^{n-m}\right)= \begin{cases}1 & \text { if }[\lambda]_{k}^{n-m}=\left[\lambda_{i}-\lambda_{j}\right]^{n-m}, \\ 0 & \text { otherwise }\end{cases}
$$

then the following recursion relation holds,

$$
d_{j k}^{n}=\sum_{j=1}^{x} H\left([\lambda]_{k}^{n},[\lambda]_{j^{\prime}}^{n-\tilde{\lambda}_{1 j}} ;\left[\tilde{1}^{\tilde{\lambda}_{1 j}}\right]\right) d_{x j^{\prime}}^{n-\tilde{\lambda}_{1 j}},
$$

where $x$ is the index of the partition of $n-\tilde{\lambda}_{1 j}$ obtained by removing the first column of partition number $j$. If $x$ labels a one-or two-columned pattern, the recursive process has come to an end since then clearly $d_{x j^{3}}^{n-x_{1 j}}=1$.

For most applications in nuclear physics where the space symmetry is restricted to patterns of four columns or less the calculation of $d_{j k}^{n}$ using (A10) will require at most two steps.

${ }^{1}$ K. T. Hecht, Ann. Rev. Nucl. Sci. 23, 123 (1973).

${ }^{2} \mathrm{M}$. Hamermesh, Group Theory (Addison-Wesley, Reading, Mass., 1964), Chap. 10.

${ }^{3} \mathrm{~B} . \mathrm{G}$. Wybourne, Symmetry Principles in Atomic Spectroscopy (Addison-Wesley, Reading, Mass., 1964); E. M.

Ibrahim, Roy. Soc. Dep. of Math. Tables, No. 1 (1950);

D.E. Littlewood, J. Lond. Math. Soc. 11, 49 (1936); R.

Perez and J.Flores, Nucl. Data Tables A 4, 265 (1968).

${ }^{4} \mathrm{~K} . \mathrm{V}$. Krishna Brahmam and R.D. Ratna Raju, Atomic and Nucl. Data Tables 16, 165 (1975).

${ }^{5}$ V. K. B. Kota, J. Phys. A 10, L39 (1977).

${ }^{6}$ Univ. of Michigan internal report No. 257.

${ }^{2} \mathrm{D} . \mathrm{E}$. Littlewood, The Theory of Group Characters and Matrix Representations of Groups (Oxford U.P., Oxford, 1940).

${ }^{8}$ W. J. Holman and L. C. Biedenharn, in Group Theory and its Applications, edited by E. M. Loebl (Academic, New York, 1971), Chap. 1.

${ }^{9}$ J. P. Elliot, Proc. Roy. Soc. A 245, 128 (1958). 